\title{
Fibulin-5 is a Prognostic Marker that Contributes to Proliferation and Invasion of Human Glioma Cells
}

\author{
Xu-Dong Sheng ${ }^{1}$,Hu Chen ${ }^{2}$,Hui Wang ${ }^{1}$, Zhi-Bin Ding ${ }^{1}$, Gang-Zhu Xu ${ }^{1}$, Jun-Feng \\ Zhang ${ }^{1}$, Wen-Chao $\mathrm{Lu}^{1}$, Tao $\mathrm{Wu}^{1}$, Ling $\mathrm{Zhao}^{3 *}$
}

\begin{abstract}
Fibulin-5 has recently been considered as a potential tumor suppressor in human cancers. Several studies have shown that it is down-regulated in a variety of tumor types and inhibits tumor growth and metastasis. This study was aimed to investigate the clinical significance of fibulin-5 in glioma and its role in cell proliferation and invasion. We found that the expression of fibulin-5 in glioma tissues was significantly lower than those in normal brain (NB) tissues. Negative expression was significantly correlated with advanced clinical stage (grade III+IV) . Furthermore, Fibulin-5 negative expression was correlated with a shorter overall survival of glioma patients. Multivariate Cox repression analysis indicated that fibulin-5 was an independent factor for predicting overall survival of glioma patients. Overexpression obviously inhibited cell proliferation in U251 and U87 cells. Furthermore, it significantly reduced the number of migrating and invading glioma cells. In conclusion, impaired expression of fibulin- 5 is correlated with the advanced tumor stage in glioma. Otherwise, Fibulin-5 is an independent prognostic marker for predicting overall survival of glioma patients. Mechanistically, it may function as a tumor suppressor via inhibiting cell proliferation and invasion in gliomas.
\end{abstract}

Keywords: Fibulin-5 - glioma - prognosis - proliferation - metastasis

Asian Pac J Cancer Prev, 16 (2), 769-773

\section{Introduction}

Malignant glioma is the commonest malignant tumor in brain (Li et al., 2014b). World health organization (WHO) classification system is used for tumor grading and has implications for prognosis and management (Brat et al., 2007; Gu et al., 2014). Tumor resection followed by radiotherapy and temozolomide treatment is the current standard therapy for glioma. But most of glioma patients exhibit tumor progression within two years after diagnosis (Hagemann et al., 2013). Thus, it is important to develop a potent prognostic marker and therapeutic target for human glioma.

The Fibulins is an old protein family, which is conserved in both worms and humans (Hwang et al., 2013). Fibulins are structurally consisting with a globular Fibulin-type module in C-terminal and calcium-binding epidermal growth factor (EGF)-like modules (Hwang et al., 2013). In mammalian, the Fibulin family contains seven members, which were described as Fibulins 1-7. It has been reported that Fibulin gene mutations are related to various human diseases (Argraves et al., 2003; Noda et al., 2013). The Fibulins stabilize the structures of the extra cellular matrix (ECM) by interacting with many kinds of ECM components, such as laminin, elastin, aggrecan, endostatin and fibronectin (Nakamura et al.,
2002; Schiemann et al., 2002; Papke and Yanagisawa, 2014). Otherwise, Fibulins are involved in fibrogenesis, tissue organogenesis, vasculogenesis and tumorigenesis (Schiemann et al., 2002; de Vega et al., 2009). Fibulin-5 overexpression triggers DNA synthesis and stimulates motility in fibrosarcoma, fibroblasts and breast cancer cells (Hwang et al., 2013). Nevertheless, the expression of Fibulin-5 is impaired in various human cancer tissues including renal cell carcinoma, bladder urothelial carcinoma, prostate cancer, lung cancer, colorectal cancer and hepatocellular carcinoma (Wlazlinski et al., 2007; Yanagisawa et al., 2009; Hu et al., 2011; Ohara et al., 2011; Tu et al., 2014). But the clinical significance of Fibulin-5 and its function in human glioma is poorly understood.

In this study, we demonstrate that reduced expression of Fibulin-5 is correlated with advanced tumor stage in glioma. The expression of Fibulin-5 is an independent prognostic marker for predicting overall survival of glioma patients. Furthermore, we disclose that Fibulin-5 may inhibit tumour progression by suppressing cell proliferation and invasion in human glioma.

\section{Materials and Methods}

\section{Clinical samples}

A total of 86 paraffin-embedded glioma and 20

${ }^{1}$ Department of Neurosurgery, ${ }^{3}$ Department of Anesthesiology, the First Affiliated Hospital of Xi' an Medical College, ${ }^{2}$ Department of Neurosurgery, Tang-Du Hospital, Fourth Military Medical University, Xi'an, China *For correspondence:jgsy_2014@sina.com 
normal brain (NB) tissues samples were obtained from the Department of Neurosurgery, Tang-Du Hospital of the Fourth Military Medical University during January 2008 to December 2010. All samples were used after obtaining informed consent. The demographic features and clinicopathologic date are shown in Table 1. All specimens had confirmed pathological diagnosis and were classified according to the WHO criteria.

The Xi'an Medical College Ethics Committee approved all protocols according to the Helsinki Declaration (as revised in Edinburgh 2000).

\section{Immunohistochemical staining}

Immunohistochemistry with streptavidin peroxidase conjugated (SP-IHC) method was performed on formalinfixed paraffin sections. Sections that were underwent dewaxed, rehydration, antigen retrieval, endogenous peroxidase activity blocking and goat serum blocking were incubated with Fibulin-5 (R\&D system, Minneapolis, MN, USA) antibody at $4{ }^{\circ} \mathrm{C}$ overnight. SP conjugated secondary antibody and diaminobenzidine (DAB) were used for staining of sections. According to the percentage of positive tumor cells, Fibulin-5 expression was classified as negative expression (less than $10 \%$ ) and positive expression (equal and more than 10\%) (Tu et al., 2014). Real time quantitative reverse transcription-PCR (qRTPCR) Fibulin-5 primers (forward: 5'-TCG CTA TGG TTA CTG CCA GCA-3'; reverse: 5'-TTG GCA AGA CCT TCC ATC GTC-3'). The PCR amplification for the quantification of the Fibulin-5 mRNA and the GAPDH mRNA was performed using a SYBR ${ }^{\circledR}$ Premix Ex Taq ${ }^{\text {TM }}$ ii (Perfect Real Time) Kit (Takara Bio, Shiga, Japan), as previously described (Tu et al., 2014).

\section{Cell line and transfection}

The human glioma cell lines, U251 and U87 (Chinese Academy of Sciences, Shanghai, China), were cultured in complete Dulbecco's modified Eagle medium (DMEM, Gibco, Grand Island, NY, USA) containing 10\% fetal bovine serum (FBS, Gibco) with 100 units/mL penicillin and $100 \mu \mathrm{g} / \mathrm{mL}$ streptomycin (Sigma, St-Louis, MO, USA) in a humidified containing of $5 \% \mathrm{CO} 2$ incubator at $37^{\circ} \mathrm{C}$. Expression vector pMMP-Fibulin-5 was generated by inserting the cDNA into pMMP. Cells were transfected with the vector mentioned above using Lipofectamine 2000 according to the manufacturer's instructions (Invitrogen, Carlsbad, CA, USA).

\section{Western blotting}

Fibulin-5 (R\&D system, Minneapolis, MN, USA) and GAPDH (G8140, US Biological, Salem, MA, USA) antibodies were used for immunoblotting assay. Horseradish peroxidase (HRP)-conjugated secondary antibody (Bio-Rad, Hercules, CA, USA) were used at a dilution from 1:1000-1:5000 and detected by a Western Blotting Luminol Reagent (sc-2048, Santa Cruz, CA, USA).

\section{BrdU cell proliferation assay}

For the proliferation assay, glioma cells that transfected with pMMP-Fibulin-5 or empty vector (EV) were seeded into 96-well plates at 5000 cells/well for 24 hours and assessed using a Cell Proliferation ELISA, BrdU (5-bromodeoxyuridine) (chemiluminescent) (Roche, Indianapolis, IN, USA).

\section{Transwell cell migration and invasion assays}

Transwell cell migration assays were done in 12 well plates with $8-\mu \mathrm{m}$ BioCoat control inserts (Becton Dickinson Labware, Bedford, MA). 1-2 × 105 pMMPFibulin-5 or EV transfected U251 or U87 cells that were suspended in $500 \mu \mathrm{L}$ serum free DMEM were seeded in the upper well and DMEM medium with $10 \%$ FBS, as indicated, in the lower well. After completion, membranes were removed, wiped on the side facing the upper well, and stained with crystal violet. At least 6 representative images of each well were taken and cell numbers were counted using ImageJ. BioCoat Matrigel invasion chamber (Becton Dickinson Labware) was used for transwell cell invasion assays and the following protocols were the same as transwell cell migration assays (Li et al., 2014a).

The experiments were all performed in triplicate for confirmation.

\section{Statistical analysis}

Results are expressed as Mean \pm SEM. Significance was established, with the SPSS statistical package for Windows Version 13 (SPSS, Chicago, IL, USA) or GraphPad Prism 5 software (GraphPad Software, Inc, San Diego, CA, USA), using a Pearson chi-squared test, a Kaplan-Meier plot, a log-rank test, the multi-variant Cox regression analysis or a two-tailed Student's t test, when appropriate.

Differences were only considered significant when $p<0.05$.

Table 1. Correlation between the Clinicopathologic Characteristics and Expression of Fibulin-5 Protein in Glioma

\begin{tabular}{|c|c|c|c|c|c|}
\hline \multirow[t]{2}{*}{ Characteristics } & & \multirow[t]{2}{*}{ Total No. of patients, $n=86$} & \multicolumn{2}{|c|}{ No. of patients } & \multirow[t]{2}{*}{$p$} \\
\hline & & & Fibulin-5 negative & Fibulin-5 positive & \\
\hline \multirow[t]{2}{*}{ Age (y) } & $<50$ & 40 & 29 & 11 & 0.468 \\
\hline & $\geq 50$ & 46 & 30 & 16 & \\
\hline \multirow[t]{2}{*}{ Sex } & Male & 55 & 38 & 17 & 0.897 \\
\hline & Female & 31 & 21 & 10 & \\
\hline \multirow[t]{3}{*}{ Histologic type } & Astrocytic tumors & 62 & 42 & 20 & 0.153 \\
\hline & Oligodendrogial tumors & 9 & 7 & 2 & \\
\hline & Oligoastrocytic tumors & 15 & 10 & 5 & \\
\hline \multirow[t]{2}{*}{ WHO grade } & $\mathrm{I}+\mathrm{II}$ & 26 & 12 & 14 & $0.003 *$ \\
\hline & III+IV & 60 & 47 & 13 & \\
\hline
\end{tabular}

WHO, World Health Organization; *Statistically significant 


\section{Results}

Expression of Fibulin-5 in glioma and NB tissues

To determine the expression of Fibulin-5 in glioma specimens, we detected the levels of Fibulin-5 expression in 86 collected glioma tissues and 20 collected NB tissues using immunohistochemical staining. The Fibulin-5 expression was considered as either negative or positive. Our data indicated that Fibulin-5 positive expression was observed in $80.0 \%$ (16/20) of NB tissues, while only $31.4 \%(27 / 86)$ of glioma tissues showed a positive Fibulin-5 signal ( $p<0.05$, Figure 1$)$. These data indicate that the level of Fibulin-5 in glioma tissues was significantly lower as compared with that in NB tissues.

\section{Clinical significance of Fibulin-5 in glioma cases}

To investigate the clinical significance of Fibulin-5 in glioma, we analyzed the correlation between Fibulin- 5 expression and clinicopathological parameters in glioma. As shown in Table1, Clinical association analysis using a Pearson chi-squared test indicated that Fibulin-5 negative expression was evidently correlated with advanced clinical



Figure 1. Immunohistochemical staining of Fibulin-5 in glioma and NB tissues. Fibulin-5 was localized within the cytoplasm. Low (B), medium (C) and high (D) expression of Fibulin-5 in the tumor cells of glioma tissue compared to NB tissues with positive staining (A). Scale bar: $100 \mu \mathrm{m}$

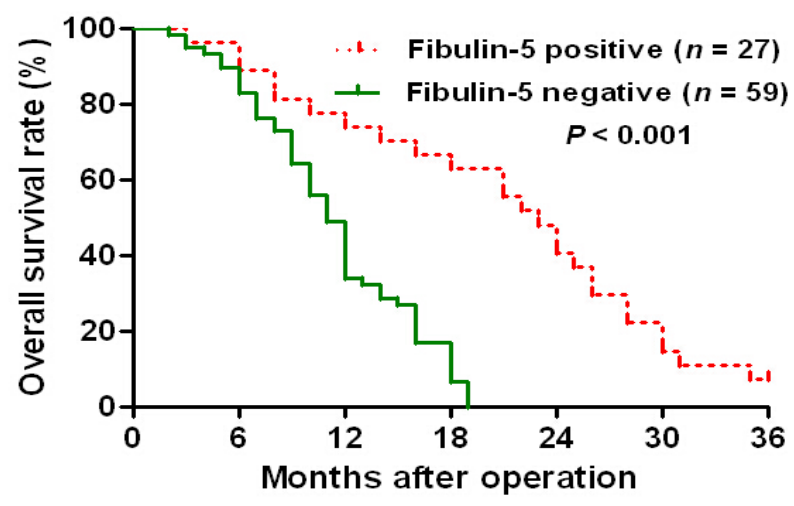

Figure 2. Kaplan-meier Survival Analysis of Overall Survival in 86 Glioma Patients according to Fibulin-5 Protein Expression. The negative expression of Fibulin-5 conferred an unfavorable survival for glioma patients stage (grade III+IV; $p=0.003$ ). Furthermore, Kaplan Meier estimation indicated that tumors with Fibulin-5 negative expression indeed associated with a shorter overall survival of glioma patients ( $p<0.05$, Figure 2$)$. Importantly, Fibulin-5 expression was an independent factor for predicting overall survival of glioma patients ( $p=0.018$, Table 2$)$. These data indicate that Fibulin-5 may act as a potent biomarker for predicting prognosis of glioma patients.

\section{Fibulin-5 overexpression suppresses cell proliferation in glioma cells}

To confirm the role of Fibulin-5 in glioma, U251 and U87 cells that were transduced with pMMP-Fibulin-5 or empty vector $(\mathrm{EV})$ were subjected to BrdU incorporation assays for cell proliferation. As measured by Western blot, the level of Fibulin-5 protein was significantly up-regulated by exogenous vector transfection in both U251 and U87 cells (Figure 3A). BrdU incorporation assays were performed to determine the effect of altering Fibulin-5 levels on tumor cell proliferation. We found that Fibulin-5 overexpression resulted in a significant reduction of both $\mathrm{U} 251$ and $\mathrm{U} 87$ cell proliferation $(p<0.05$, respectively; Figure 3B).

Table 2. Multivariate Cox Regression Analysis Of Overall Survival Duration

\begin{tabular}{lccc}
\hline Variables & \multicolumn{3}{c}{ Multivariate analysis } \\
& HR & $95 \%$ CI & $p$ \\
\hline Age & 2.113 & $0.888-5.030$ & 0.091 \\
Sex & 0.749 & $0.339-1.652$ & 0.474 \\
Histologic type & 1.846 & $0.694-4.908$ & 0.219 \\
WHO grade & 3.576 & $1.251-10.211$ & $0.017^{*}$ \\
Fibulin-5 expression & 0.253 & $0.081-0.790$ & $0.018^{*}$ \\
\hline
\end{tabular}

WHO, world health organization; HR, hazard ratio; CI, confidence interval. *Statistically significant
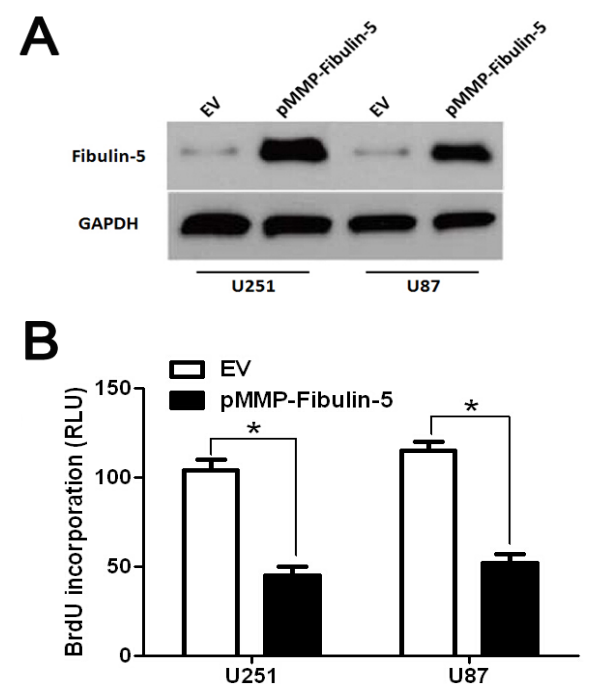

Figure 3. Fibulin-5 Overexpression Reduces Cell Proliferation in Glioma Cells. A) U251 and U87 cells that were transfected with pMMP-Fibulin-5 or empty vector (EV) were subjected to Western blot for Fibulin-5. The data are representative of multiple independent experiments. B) Cell proliferation as measured by $\mathrm{BrdU}$ incorporation assays was inhibited by Fibulin- 5 overexpression in U251 and U87 cells as compared with control cells. $\mathrm{n}=6,{ }^{*} p<0.05$ by t test 

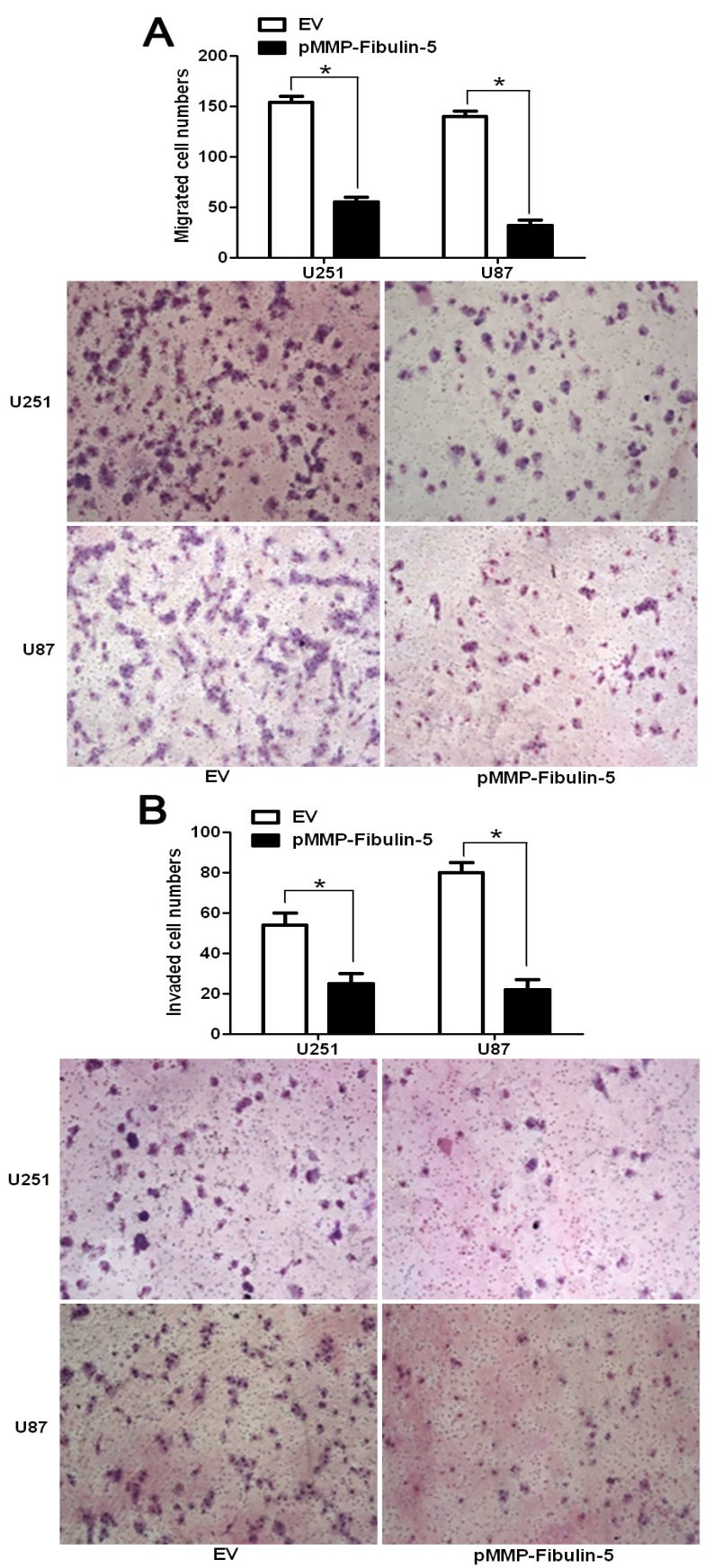

Figure 4. Fibulin-5 Overexpression Inhibits Cell Migration and Invasion in Glioma Cells. A) Cell migration as measured by Transwell assays was inhibited by Fibulin-5 overexpression in U251 and U87 cells as compared with control cells. $\mathrm{n}=6, * p<0.05$ by t test. B) Fibulin-5 overexpressing U215 and U87 cells conferred a less number of invaded cells as compared with control cells. $\mathrm{n}=6,{ }^{*} p<0.05$ by t test

Fibulin-5 overexpression inhibits glioma cell migration and invasion

Next, U251 and U87 cells that were transduced with pMMP-Fibulin-5 or empty vector (EV) were subjected to Transwell assays for cell migration and invasion. We found that Fibulin-5 overexpression resulted in a significant reduction of cell migration in both $\mathrm{U} 251$ and U87 cells ( $p<0.05$, respectively; Figure 4A). Furthermore, the number of invaded glioma cells was prominently decreased after Fibulin-5 overexpression $(p<0.05$, respectively; Figure 4B). Taken together, Fibulin-5 may exert an anti-cancer effect by inhibiting cell proliferation and mobility in glioma.

\section{Discussion}

The ECM protein Fibulin-5, a transforming growth factor (TGF)- $\beta$-induced glycoprotein, is a multifunctional molecule that regulates various cellular processes, including proliferation, motility and invasion, in normal and malignant cells (Hu et al., 2011; Li et al., 2012; Xiao et al., 2013). Reduced expression of Fibulin-5 has been observed in a number of human cancers (Schiemann et al., 2002; Wlazlinski et al., 2007; Yanagisawa et al., 2009; Hu et al., 2011; Ohara et al., 2011). In this study, the expression of Fibulin-5 was detected in 86 glioma tissues and 20 NB tissues using immunohistochemical staining. We found that Fibulin-5 protein expression in the glioma tissues was prominently lower than that in the NB tissues. Clinical analysis showed that the negative expression of Fibulin-5 was obviously correlated with advanced clinical stage in glioma. Importantly, our data showed that Fibulin-5 negative expression conferred a significant shorter overall survival for glioma patients. Multivariate Cox repression analysis indicated that Fibulin-5 was an independent factor for predicting overall survival of glioma patients. Taken together, our results indicate that Fibulin-5 may be a potential tumor suppressor and act as a prognostic biomarker for predicting survival of glioma patients.

Fibulin-5 expression may be associated with the suppression of tumor formation through its control of cell proliferation, motility and angiogenic sprouting (Albig and Schiemann, 2005). However, what is the role of Fibulin-5 in glioma? Here, we disclosed new role of Fibulin-5 in glioma. Fibulin-5 was overexpressed in U251 and U87 cells via exogenous vector transfection. BrdU incorporation assays demonstrated that Fibulin-5 overexpression obviously inhibited cell proliferation in U251 and U87 cells. Furthermore, Transwell migration assays found that Fibulin-5 overexpression led to a significant reduction of cell migration in both U251 and U87 cells. Otherwise, Transwell invasion assays showed that Fibulin-5 overexpression decreased the number of invaded glioma cells. Our data indicate that Fibulin-5 may inhibit tumour progression by suppressing cell growth and mobility in glioma.

In conclusion, we find that the expression of Fibulin-5 is reduced in glioma tissues and its negative expression is correlated with advanced tumor stage. Furthermore, Fibulin-5 is an independent prognostic marker for predicting overall survival of glioma patients. Fibulin-5 overexpression reduces cell proliferation and decreases the number of migrated and invaded glioma cells in vitro. Altogether, Fibulin-5 may be a potential valuable biomarker and therapeutic target for human glioma.

\section{References}

Albig AR, Schiemann WP (2005). Fibulin-5 function during tumorigenesis. Future Oncol, 1, 23-35.

Argraves WS, Greene LM, Cooley MA, et al (2003). Fibulins: 
physiological and disease perspectives. EMBO Rep, $\mathbf{4}$, 1127-31.

Brat DJ, Scheithauer BW, Fuller GN, et al (2007). Newly codified glial neoplasms of the 2007 WHO Classification of Tumours of the Central Nervous System: angiocentric glioma, pilomyxoid astrocytoma and pituicytoma. Brain Pathol, 17, 319-24.

de Vega S, Iwamoto T, Yamada Y (2009). Fibulins: multiple roles in matrix structures and tissue functions. Cell Mol Life Sci, 66, 1890-902.

Gu WT, Gu SX, Shou JJ (2014). Pathway and network analysis in glioma with the partial least squares method. Asian Pac J Cancer Prev, 15, 3145-9.

Hagemann C, Fuchs S, Monoranu CM, et al (2013). Impact of MACC1 on human malignant glioma progression and patients' unfavorable prognosis. Neuro Oncol, 15, 1696-709.

$\mathrm{Hu} \mathrm{Z}$, Ai Q, Xu H, et al (2011). Fibulin-5 is down-regulated in urothelial carcinoma of bladder and inhibits growth and invasion of human bladder cancer cell line 5637. Urol Oncol, 29, 430-5.

Hwang CF, Shiu LY, Su LJ, et al (2013). Oncogenic fibulin-5 promotes nasopharyngeal carcinoma cell metastasis through the FLJ10540/AKT pathway and correlates with poor prognosis. PLoS One, $\mathbf{8}, 84218$.

Li C, Yang W, Zhang J, et al (2014a). SREBP-1 has a prognostic role and contributes to invasion and metastasis in human hepatocellular carcinoma. Int J Mol Sci, 15, 7124-38.

Li F, Xu H, Zeng Y, et al (2012). Overexpression of fibulin-5 in retinal pigment epithelial cells inhibits cell proliferation and migration and downregulates VEGF, CXCR4, and TGFB1 expression in cocultured choroidal endothelial cells. Curr Eye Res, 37, 540-8.

Li PD, Wang XJ, Shan Q, et al (2014b). Evaluation of TAZ expression and its effect on tumor invasion and metastasis in human glioma. Asian Pac J Trop Med, 7, 757-60.

Nakamura T, Lozano PR, Ikeda Y, et al (2002). Fibulin-5/ DANCE is essential for elastogenesis in vivo. Nature, $\mathbf{4 1 5}$, 171-5.

Noda K, Dabovic B, Takagi K, et al (2013). Latent TGF-beta binding protein 4 promotes elastic fiber assembly by interacting with fibulin-5. Proc Natl Acad Sci USA, 110, 2852-7.

Ohara H, Akatsuka S, Nagai H, et al (2011). Stage-specific roles of fibulin-5 during oxidative stress-induced renal carcinogenesis in rats. Free Radic Res, 45, 211-20.

Papke CL, Yanagisawa H (2014). Fibulin-4 and fibulin-5 in elastogenesis and beyond: Insights from mouse and human studies. Matrix Biol, 37, 142-9.

Schiemann WP, Blobe GC, Kalume DE, et al (2002). Contextspecific effects of fibulin-5 (DANCE/EVEC) on cell proliferation, motility, and invasion. Fibulin-5 is induced by transforming growth factor-beta and affects protein kinase cascades. J Biol Chem, 277, 27367-77.

Tu K, Dou C, Zheng X, et al (2014). Fibulin-5 inhibits hepatocellular carcinoma cell migration and invasion by down-regulating matrix metalloproteinase-7 expression. BMC Cancer, 14, 938 .

Wlazlinski A, Engers R, Hoffmann MJ, et al (2007). Downregulation of several fibulin genes in prostate cancer. Prostate, 67, 1770-80.

Xiao W, Zhou S, Xu H, et al (2013). Nogo-B promotes the epithelial-mesenchymal transition in HeLa cervical cancer cells via Fibulin-5. Oncol Rep, 29, 109-16.

Yanagisawa H, Schluterman MK, Brekken RA (2009). Fibulin-5, an integrin-binding matricellular protein: its function in development and disease. J Cell Commun Signal, 3, 337-47. 